\title{
Dispersive techniques for low-energy QCD: Pion-pion and pion-kaon scattering
}

\author{
Jacobo Ruiz de Elvira* \\ Albert Einstein Center for Fundamental Physics, \\ Institute for Theoretical Physics, University of Bern, \\ Sidlerstrasse 5, CH-3012 Bern, Switzerland \\ E-mail: elvira@itp.unibe.ch
}

\begin{abstract}
A good understanding of strong interactions at low energies is key in the precision frontier of Particle and Hadron Physics, since most hadronic observables end up in final states involving pions and kaons, the lightest mesons in the Hadron Spectrum. In particular, the pion-pion and pion-kaon scattering lengths are the lowest energy observables for these interactions, and hence a fundamental quantity for understanding hadron interaction at low energies. In this talk we review the current status of their determination. After discussing the predictions expected from chiral symmetry at different orders in the chiral expansion, we review current experimental and lattice determinations. We then focus on the dispersive determination of pion-pion and pion-kaon interactions, based on the analysis of Roy and Roy-Steiner equation, and continue discussing in detail the current tension between the chiral symmetry and dispersive predicition for the pionkaon scattering lenthgs. We finish this talk providing an explanation of this disagreement.
\end{abstract}

The 9th International Workshop on Chiral Dynamics

17-21 September 2018

Durham, NC, USA

\footnotetext{
*These proceedings draw from a previous conference contribution [1].
} 


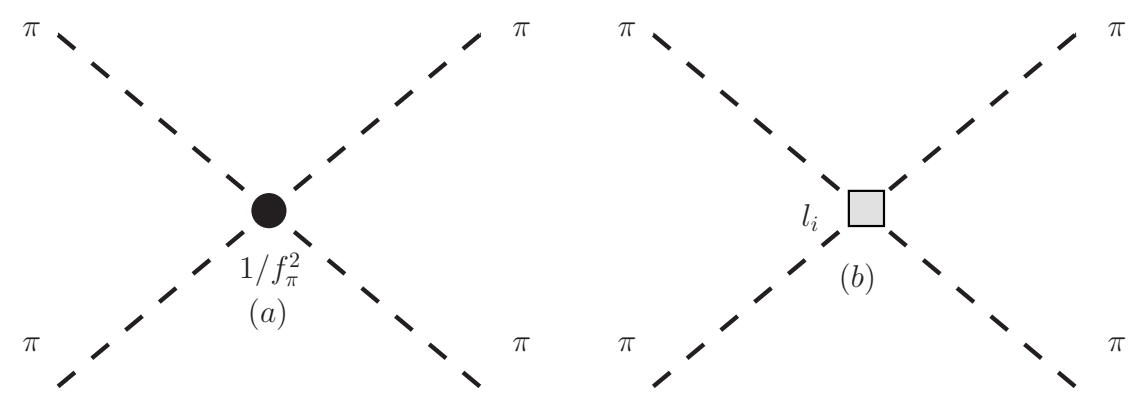

Figure 1: (a) Leading-order diagram for $\pi \pi$ scattering in chiral perturbation theory. (b) Next-to-leadingorder diagram depending on the low-energy constants $l_{1-4}$.

\section{Introduction}

Pion-Pion and pion-kaon scattering are the simplest processes to test our understanding of the chiral symmetry-breaking pattern for the light- and strange-quark sector of $\mathrm{QCD}$, respectively. In particular, their low-energy parameters, most notably the scattering lengths, encode relevant information about the spontaneous and explicit chiral symmetry breaking. Being low-energy observables, their properties can be efficiently studied using the effective field theory of Quantum Chromodynamics (QCD) at low energies, Chiral Perturbation Theory (ChPT) [2-4], constructed as a systematic expansion around the chiral limit of QCD in terms of momenta and quark masses.

Thanks to isospin, crossing and Bose-Einstein symmetries, $\pi \pi$ scattering can be expressed in terms of only three independent amplitudes with well defined isospin $I$ in the $s$-channel, namely

$$
\begin{aligned}
& T_{I=0}(s, t, u)=3 A(s, t, u)+A(t, s, u)+A(u, t, s), \\
& T_{I=1}(s, t, u)=A(t, s, u)-A(u, t, s), \\
& T_{I=2}(s, t, u)=A(t, s, u)+A(u, t, s),
\end{aligned}
$$

where $A(s, t, u)$ stands for the $\pi^{+}\left(p_{1}\right) \pi^{-}\left(p_{2}\right) \rightarrow \pi^{0}\left(p_{1}^{\prime}\right) \pi^{0}\left(p_{2}^{\prime}\right)$ amplitude and $s=\left(p_{1}+p_{2}\right)^{2}, t=\left(p_{1}-\right.$ $\left.p_{1}^{\prime}\right)^{2}$ and $u=\left(p_{1}-p_{2}^{\prime}\right)^{2}$ are the standard choice of Mandelstam variables.

At leading order (LO) in la chiral expansion, i.e., in the expansion of the pion mass and momenta, the $\pi \pi$ scattering amplitude is given by the Feynman diagram shown in Fig. 1a, resulting in the well-known low-energy theorems for the $S$-wave scattering lengths, the amplitudes evaluated at threshold $[3,5]$ :

$$
a_{0}^{0}=\frac{7 m_{\pi}^{2}}{32 \pi f_{\pi}^{2}}+O\left(m_{\pi}^{4}\right)=0.16+\ldots, \quad a_{2}^{0}=-\frac{m_{\pi}^{2}}{16 \pi f_{\pi}^{2}}+O\left(m_{\pi}^{4}\right)=-0.045+\ldots
$$

where the numerical values are given in $m_{\pi}$ units. The scattering lengths are hence predicted solely in terms of the pion mass and decay constant $f_{\pi}$, showing that, at threshold, the interaction is attractive for the isoscalar $I=0$ channel and repulsive for the isotensor $I=2$ one. In addition, the S-wave scattering lengths are both proportional to $m_{\pi}$, so that the interaction vanishes in the chiral limit. The light value of the physical pion mass highlights that pions interact indeed very weakly at low energies and it suggests a fast convergence of the chiral series for the scattering lengths. In 
fact, from the chiral counting one naively expects higher order corrections to be

$$
\left.\left.a_{J}^{I}\right|_{N^{n} L O} \propto a_{J}^{I}\right|_{L O}\left(\frac{m_{\pi}}{4 \pi f_{\pi}}\right)^{2 n}, \quad \text { with } \quad n>1,
$$

The pion-pion scattering amplitude was derived and studied at next-to-leading order (NLO) in $[3,4]$ and at next-to-next-leading order (NNLO) in [6,7]. Higher order corrections involve loop diagrams, which might generate large contributions. Nevertheless, they are suppressed at threshold and hence their role for the pion-pion scattering lengths is expected to be relatively small. In addition, the $\pi \pi$ scattering amplitude depends at higher orders on a list of low-energy constants (LECs), which, encoding information about heavier degrees of freedom, can not be constrained from chiral symmetry solely, Fig. 1b. Once determined in one process, these LECs can subsequently be used to predict others. Using LECs estimates from [20] one obtains for the pion-pion scattering lengths

$$
\begin{aligned}
& a_{0}^{0}=\underbrace{0.156}_{L O}+\underbrace{0.044}_{N L O}+\underbrace{0.017}_{N N L O}+\cdots=0.217, \\
& a_{0}^{2}=\underbrace{-0.045}_{L O}+\underbrace{0.002}_{N L O} \underbrace{0.001}_{N N L O}+\cdots=-0.042 .
\end{aligned}
$$

Whereas the value of the isotensor scattering length changes from one order to the next one consistently with the chiral counting prediction in (1.3), higher-order contribution for the isoscalar scattering length are larger than expected. Namely, NLO and NNLO corrections increase $a_{0}^{0}$ by $28 \%$ and $11 \%$, respectively. The reason of these large corrections are very well known: the scalar isoscalar pion-pion channel suffers from strong final state interactions that produce a large curvature at threshold and increase the scattering length value. This curvature is indeed reflected in the quark mass expansion of $a_{0}^{0}$

$$
a_{0}^{0}=\frac{7 m_{\pi}^{2}}{32 \pi F_{\pi}^{2}}\left(1-\frac{9 m_{\pi}^{2}}{32 \pi^{2} F_{\pi}^{2}} \log \left(\frac{m_{\pi}^{2}}{\mu^{2}}\right)+\cdots\right),
$$

which shows a coefficient for the chiral logarithm nine times larger than the leading term. This slow convergence points out that while the expansion on the pion mass is guaranteed by the small value of $m_{\pi}$, the expansion in momenta does not converge equally well in the whole low-energy region. This is one of the main motivations to consider dispersion theory, which provides a much efficient control of the energy dependence of low-energy scattering processes.

The pion-pion scattering lengths are also well determined from the experimental side, where $K_{l 3}$ and $K_{l 4}$ decays provide a strong constraint for their values. The most accurate experimental determination currently comes from the NA48/2 experiment at CERN [9]

$$
a_{0}^{0}=(0.222 \pm 0.014), \quad a_{2}^{0}=(-0.0432 \pm 0.0097),
$$

fully compatible with the NNLO ChPT predictions. On the lattice side, the isotensor pion-pion scattering length is also well determined by several collaborations (see for instance [10] and references therein). Nevertheless, the isoscalar scattering length remains difficult due to large disconnected diagram contributions and at present there are only two independent unquenched results $[11,12]$. Finally, the most precise determinations for the $\pi \pi$ scattering come from dispersion theory, where 


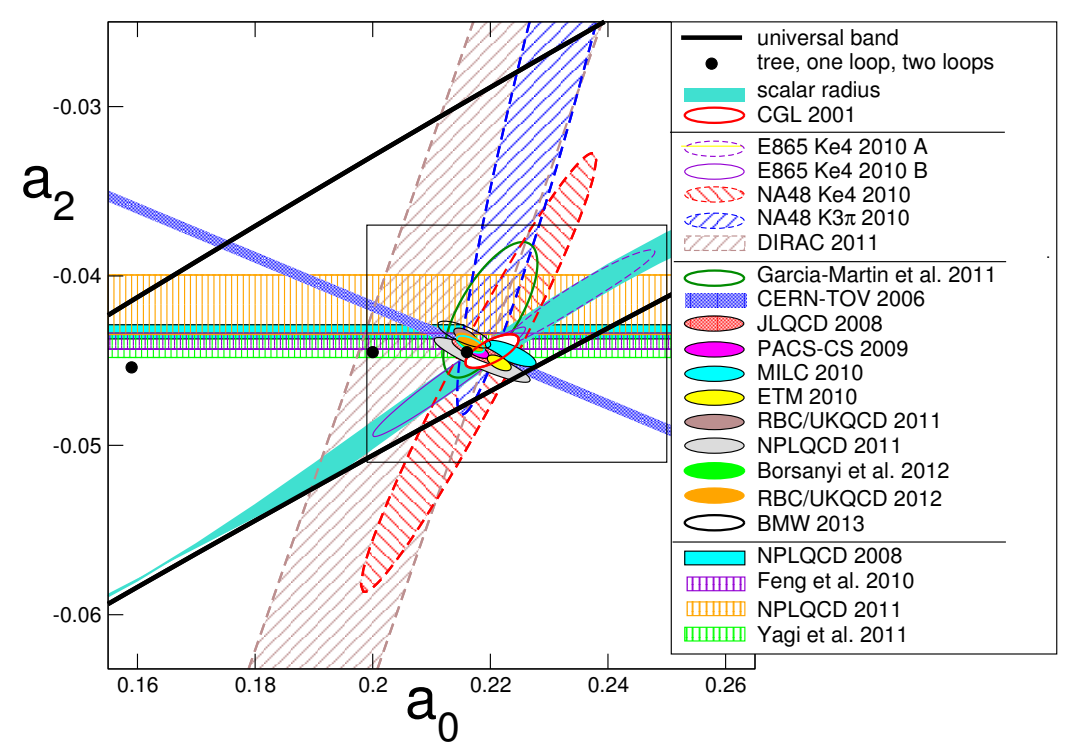

Figure 2: Different experimental, phenomenological and lattice QCD determination of the pion-pion scattering lengths. The chiral prediction at LO, NLO and NNLO are denoted by a black dot. Figure courtesy of H. Leutwyler.

Roy-equation analyses provide [13-16]

$$
\begin{array}{lll}
a_{0}^{0}=(0.220 \pm 0.005), & a_{2}^{0}=(-0.0444 \pm 0.0010) & \text { from [14] } \\
a_{0}^{0}=(0.220 \pm 0.008), & a_{2}^{0}=(-0.042 \pm 0.004) & \text { from [15] }
\end{array}
$$

All ChPT, experimental, lattice and dispersive results are plotted in Fig. 2, where the $a_{0}^{0}, a_{2}^{0}$ scattering length plane is depicted. This comparison shows a consistent picture hence providing one of the most precise tests of the Standard Model at low energies.

While the situation on pion-pion kaon is extremely successful, the determination of the pionkaon scattering lengths is much less satisfactory and it will be the topic of discussion for the remaining part of this talk. Pion-kaon scattering can be expressed in terms of two independent invariant amplitudes with well defined isospin $I=1 / 2$ and $I=3 / 2$ in the $\pi K \rightarrow \pi K$ channel, namely $T^{1 / 2}$ and $T^{3 / 2}$. Nevertheless, for convenience, it is useful to combine them in terms of isospin-even and -odd amplitudes $I= \pm$, which are defined as

$$
T_{a b}=\delta_{a b} T^{+}+\frac{1}{2}\left[\tau_{a}, \tau_{b}\right] T^{-},
$$

where $a$ and $b$ denote pion isospin indices and $\tau_{a}$ stand for the Pauli matrices. Both basis are related by simple isospin transformations:

$$
T^{1 / 2}=T^{+}+2 T^{-}, \quad T^{3 / 2}=T^{+}-T^{-} .
$$

As in the pion-pion case, the $S$-wave scattering lengths at LO in the chiral expansion are given by a low-energy theorem $[4,5]$ :

$$
a_{0}^{-}=\frac{m_{\pi} m_{K}}{8 \pi\left(m_{\pi}+m_{K}\right) f_{\pi}^{2}}+O\left(m_{i}^{4}\right), \quad a_{0}^{+}=O\left(m_{i}^{4}\right)
$$




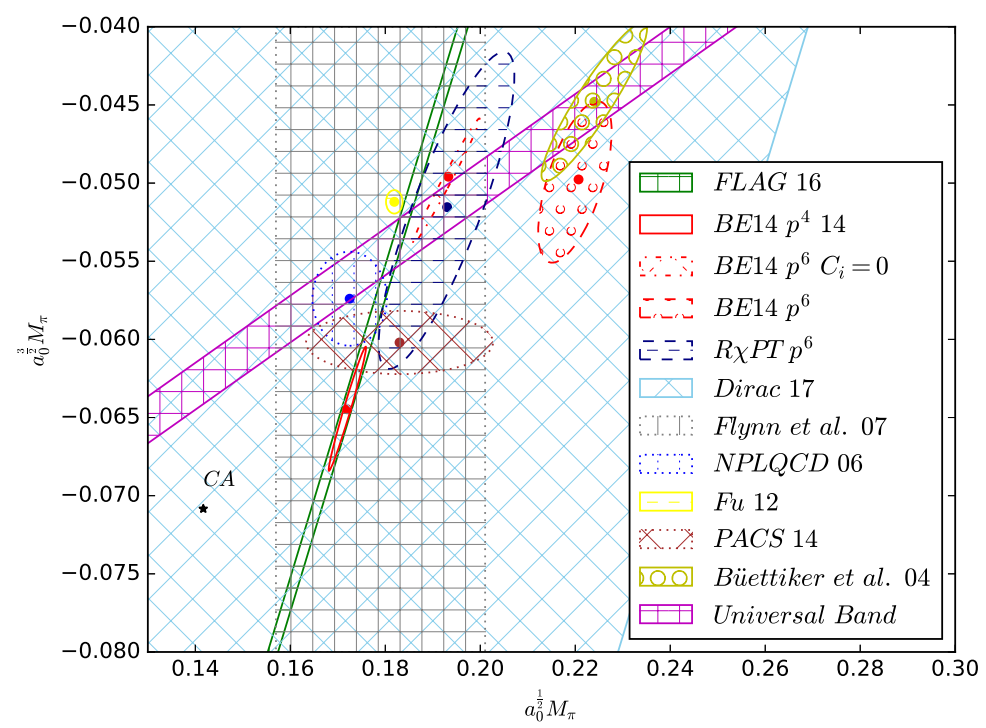

Figure 3: Different determinations of the pion-kaon scattering lengths in the $I=1 / 2, I=3 / 2$ basis. The LO ChPT value, just a result of current algebra, is denoted by a star. The NLO prediction of the isospin-odd scattering length is given by the dark-green band labelled as FLAG16. The inclusion of the isospin-even scattering length, using the LECs provided in [20], leads to the solid-red ellipse BE14 $p^{4}$. The red dotdashed ellipse, BE14 $p^{6} C_{i}=0$, corresponds to the NNLO chiral prediction when the $O\left(p^{6}\right)$ LECs are set to zero. The full NNLO result is represented by the dashed-red ellipse, albeit, as explained in the text, this result is biased to reproduce the RS dispersive values given in [22], solid-green ellipse. The NNLO results with $O\left(p^{6}\right)$ LECs estimated by resonance saturation is denoted by the dashed-blue ellipse, $R \chi P T p^{6}$. The universal band obtained from the RS analysis performed in this work is given by the violet band. The remaining experimental and lattice results are explained in the main text.

where $m_{i}$ denotes the pion $\left(m_{\pi}\right)$ or kaon $\left(m_{K}\right)$ mass. Whereas the isospin-odd scattering length is predicted once more in terms of the pion and kaon masses as well as the pion decay constant $f_{\pi}$, the isospin-even one is suppressed at low energies. The LO ChPT value for the pion-kaon scattering lengths is denoted by a star in Fig. 3, where the pion-kaon scattering length plane is plotted in the $I=1 / 2$ and $I=3 / 2$ basis.

The pion-kaon scattering amplitude at NLO was derived in $[17,18]$. The contribution from the LECs reads [17]:

$$
\begin{aligned}
& \left.a_{0}^{-}\right|_{\text {LECs }}=\frac{m_{K} m_{\pi}^{3}}{\pi\left(m_{\pi}+m_{K}\right) f_{\pi}^{4}} L_{5}+O\left(m_{i}^{6}\right), \\
& \left.a_{0}^{+}\right|_{\text {LECs }}=\frac{m_{K}^{2} m_{\pi}^{2}}{\pi\left(m_{\pi}+m_{K}\right) f_{\pi}^{4}}\left(4\left(L_{1}+L_{2}-L_{4}\right)+2 L_{3}-L_{5}+2\left(2 L_{6}+L_{8}\right)\right)+O\left(m_{i}^{6}\right) .
\end{aligned}
$$

One might wonder how stable are the NLO predictions against higher-order corrections. A pion-kaon low-energy theorem [17] imposes higher $O\left(m_{i}^{2 n}\right)$ contributions to the isospin-odd scat- 
tering length arising from contact terms to be at most:

$$
\left.\left.a_{0}^{-}\right|_{N^{n} L O} \propto a_{0}^{-}\right|_{L O}\left(\frac{m_{\pi}}{4 \pi f_{\pi}}\right)^{2}\left(\frac{m_{K}}{4 \pi f_{\pi}}\right)^{2 n} \quad \text { with } \quad n \geq 2 .
$$

Whereas the factor $\left(m_{K} / 4 \pi f_{\pi}\right) \sim 0.2$ is relatively large, the prefactor $m_{\pi}^{2} /\left(4 \pi f_{\pi}\right)^{2} \sim 0.015$ suppresses higher order corrections by roughly two orders of magnitude, i.e. the isospin-odd scattering length is protected from higher-order correction and hence one should expect small deviations from the NLO ChPT prediction for $a_{0}^{-}$.

The pion-kaon scattering amplitude at NNLO in the chiral expansion was derived in [21]. It involves a set of 32 new $O\left(p^{6}\right)$ LECs, the so called $C_{1-32}$, which unfortunately are still not well constrained from experiment. As a first step, one could estimate the size of the NNLO chiral corrections by setting all the $C_{i}$ to zero. Using for the $O\left(p^{4}\right) L_{i}$ the corresponding fit in [20], the outcome is the red dot-dashed ellipse plotted in Fig. 3. This result is consistent with our previous statement, i.e., whereas the shift between the NLO and NNLO ellipsis is small in the isovector direction, it is much larger in the isoscalar one.

The $C_{i}$ entering in pion-kaon scattering were also estimated in [20] by performing a global fit to different $\pi \pi$ and $\pi K$ observables. Nevertheless, among them, the dispersive determination of the $\pi K$ scattering lengths in [22] was used as constraint. Consequently, the full $O\left(p^{6}\right)$ results in [20] are not a genuine ChPT prediction but they are biased to satisfy the results given in [22]. The scattering length results in [20] and [22] are denoted in Fig. 3 by the dashed-red and dashed-green circle-filled ellipse, respectively. As we will discuss in detail below, the large difference one finds between the NLO and NNLO chiral estimates in the isovector direction is just a consequence of the large discrepancy between the dispersive result in [22] and chiral expectations. Alternatively, one can estimate the value of the $C_{i}$ by using resonance saturation. The contribution from vector and scalar resonances to the saturation of the $O\left(p^{6}\right)$ LECs was also studied in [21]. Using the vector and scalar resonance parameter values extracted in [23] from a global $\pi \pi$ and $\pi K$ fit, one obtains the dashed-blue ellipse in Fig. 3, which is now consistent with the NLO prediction for the isovector scattering length.

The only direct experimental information about the pion-kaon scattering lengths comes from the DIRAC experiment at CERN [24], where the lifetime of hydrogen-like $\pi K$ atoms was measured. They are a electromagnetically bound state of charged pions and kaons, $\pi^{+} K^{-}$and $\pi^{-} K^{+}$, which decay predominantly by strong interactions to the neutral pairs $\pi^{0} \bar{K}^{0}$ and $\pi^{0} K^{0}$. The $\pi K$ atom lifetime and the scattering length are related through the so-called modified Deser formula [25-27], namely

$$
\Gamma_{1 S}=8 \alpha^{3} \mu^{2} p a_{0}^{-2}\left(1+\delta_{K}\right),
$$

where $\alpha$ is the fine structure constants, $\mu$ is the reduced mass of the $\pi^{ \pm} K^{\mp}$ system, $p$ is the outgoing momentum in the center-of-mass frame and $\delta_{K}$ accounts for isospin breaking corrections [26-28]. The experimental determination of $\Gamma_{1 S}$ obtained at CERN yields [24]

$$
a_{0}^{-}=\left(0.072_{-0.020}^{+0.031}\right) m_{\pi}^{-1},
$$

which is denoted in Fig. 3 by a light-blue squared-filled band. Unfortunately, the experimental errors are still too large to provide useful information about the pion-kaon scattering lengths. Nevertheless, there is still room for improvement, the statistical precision is expected to improve by a 
factor 20 if the DIRAC collaboration manages to run its experiment using the $\mathrm{LHC} 450 \mathrm{GeV}$ proton beam.

On the lattice side, there is a plethora of results and we will only consider unquenched analyses. From a lattice analysis of the $\pi K$ scalar form factor in semileptonic $K_{l 3}$ decays, the value $a_{0}^{1 / 2}=0.179(17)(14) m_{\pi}^{-1}$ was reported in [29] for the pion-kaon scattering length in the $I=1 / 2$ channel. This value corresponds to the gray squared-filled band in Fig. 3. The first fully dynamical calculation with $N_{f}=2+1$ flavors was performed by the NPLQCD collaboration, leading to [30]

$$
a_{0}^{1 / 2}=\left(0.173_{-0.016}^{+0.003}\right) m_{\pi}^{-1}, \quad a_{0}^{3 / 2}=\left(-0.057_{-0.006}^{+0.003}\right) m_{\pi}^{-1},
$$

which is denoted in Fig. 3 by a dotted-blue ellipse. Further dynamical results for $N_{f}=2+1$ flavors were reported in [31] using a staggered-fermion formulation, $a_{0}^{1 / 2}=0.182(4) m_{\pi}^{-1}, \quad a_{0}^{3 / 2}=$ $-0.051(2) m_{\pi}^{-1}$, and by the PACS collaboration considering an improved Wilson action [32], $a_{0}^{1 / 2}=$ $0.183(18)(35) m_{\pi}^{-1}, \quad a_{0}^{3 / 2}=-0.060(3)(3) m_{\pi}^{-1}$. These results are depicted in Fig. 3 by a solidyellow and a dotted-brown ellipse, respectively.

As we have seen, all the previous results are consistent with chiral predictions, i.e. all of them are consistent within one standard deviation for the isospin-odd direction, whereas much larger differences are found in the isospin-even component. Nevertheless, the most precise up-to-date result was reported in [22] by solving a complete system of Roy-Steiner equations, corresponding to

$$
a_{0}^{1 / 2}=0.224(22) m_{\pi}^{-1}, \quad a_{0}^{3 / 2}=-0.045(8) m_{\pi}^{-1} .
$$

This result is denoted in Fig. 3 by a light-green circle-filled ellipse and it lies more than 3.5 standard deviations away from the NLO ChPT result. This disagreement is particularly puzzling in the isospin-odd direction, where the ChPT prediction is protected by the low-energy theorem given in (1.14) and one should expect NLO ChPT to provide a reasonably precise value for the pion-kaon scattering lengths. In fact, previous dispersive analyses for $\pi \pi$ scattering provided results for the scattering lengths only within a universal band $[13,14]$. High accuracy values were reached only after constraining dispersive results with chiral symmetry. Thus, one might wonder why should things be different in pion-kaon scattering.

\section{Roy-Steiner equations for $\pi K$ scattering}

Dispersion relations have repeatedly proven to be a powerful tool for studying processes at low energies with high precision. They are built upon very general principles such as Lorentz invariance, unitarity, crossing symmetry, and analyticity.

For $\pi \pi$ scattering, Roy equations (RE) [33] are obtained from a twice-subtracted fixed- $t$ dispersion relation, where the $t$-dependent subtraction constants are determined by means of $s \leftrightarrow t$ crossing symmetry, and performing a partial-wave expansion. This leads to a coupled system of partial-wave dispersion relations (PWDRs) for the $\pi \pi$ partial waves where the scattering lengthsthe only free parameters-appear as subtraction constants. The use of RE for $\pi \pi$ scattering has led to a determination of the low-energy $\pi \pi$ scattering amplitude with unprecedented accuracy [13-15], which, for the first time, allowed for a precise determination of the $f_{0}(500)$ pole parameters $[34,35]$.

In the case of $\pi K$ scattering, a full system of PWDRs has to include dispersion relations for two distinct physical processes, $\pi K \rightarrow \pi K$ ( $s$-channel) and $\pi \pi \rightarrow \bar{K} K$ ( $t$-channel), and the use of $s \leftrightarrow t$ 
crossing symmetry will intertwine $s$ - and $t$-channel equations. Roy-Steiner (RS) equations [36] are a set of PWDR that combine the $s$ - and $t$ - channel physical region by means of hyperbolic dispersion relations. The construction and solution of a complete system of RS equations for $\pi K$ scattering has been presented in [22].

In more detail, the starting point for the work in [22] is a set of fixed- $t$ dispersion for the pionkaon isospin-even and -odd scattering amplitudes, where the $t$-dependent subtraction constants are expressed in terms of hyperbolic dispersion relations passing through the threshold, i.e., where the internal and external Mandelstam variables $s$ and $u$ satisfy the condition $s \cdot u=m_{K}^{2}-m_{\pi}^{2}$. A twice- and once-subtracted version was considered for the isospin-even -odd amplitude, respectively, where the subtraction constants are the $a_{0}^{ \pm}$scattering lengths and the slope of the hyperbola in the $t$-direction for the isospin-even amplitude, $b^{+}$, which, in the end, is written in terms of a sum rule involving the $a_{0}^{-}$scattering length. Finally, the solution of the RS equations is achieved by minimizing the $\chi^{2}$-like function

$$
\chi_{\text {phys }}^{2}=\sum_{l, I_{s}} \sum_{j=1}^{N}\left(\operatorname{Re} f_{l}^{I_{s}}\left(s_{j}\right)-F\left[f_{l}^{I_{s}}\right]\left(s_{j}\right)\right)^{2},
$$

where $f_{l}^{I_{s}}$ denotes pion-kaon partial-waves with angular momentum $l$ and isospin $I_{s}, F\left[f_{l}^{I_{s}}\right]$ stands for the functional form of the RS equations for the $f_{l}^{I_{s}}$ partial wave, and the minimizing parameters are the partial waves and the pion-kaon scattering lengths. In this way, the minimum of (2.1) provides as an output the pion-kaon scattering length values given in (1.18). A relevant question is whether this solution is unique. In principle, the subtracted version built in [22] is constructed in such a way that it matches the conditions ensuring a unique RS equation solution investigated in [37]. In the $\pi \pi \mathrm{RE}$ case studied in [13], it was observed that the $\pi \pi$ scattering lengths were determined only within a universal band. Something similar was observed in the RS solution for $\pi N$ presented in [38], where precise results were obtained once the $\pi N$ scattering lengths were imposed as constraints. More precisely, the problem is connected with the number of no-cusp conditions required in order to ensure a smooth matching in the three partial waves between the dynamical solution of the RS equations and the input considered at higher energies. In [22], nocusp conditions for the $f_{0}^{1 / 2}$ and $f_{1}^{1 / 2}$ partial waves were imposed, matching precisely the number of free subtraction constants, the two pion-kaon scattering lengths $a_{0}^{ \pm}$. However, in [13] it was found for $\pi \pi$ scattering that only one no-cusp condition was enough to ensure a smooth matching, leading to a $\pi \pi$ scattering length universal band.

In order to analyze whether something similar might happen in the $\pi K$ case, we have studied further possible cusp-free RS solutions in a grid of points in the pion-kaon scattering length plane. The results are plotted in Fig. 4, where one can see that RS equation solutions for $\pi K$ scattering can be achieved within a universal band. Although the solution presented in [22] lies perfectly within this universal band, it is clearly not enough to fully constrain the values of the pion-kaon scattering lengths. As we can see in Fig. 2, this universal band is indeed consistent with both chiral predictions and the different lattice results studied above. The next step of this project will be to study whether the combination of RS equations with sum rules for subtraction constants allows one to obtain a unique and consistent solution of the $\pi K$ scattering lengths. 


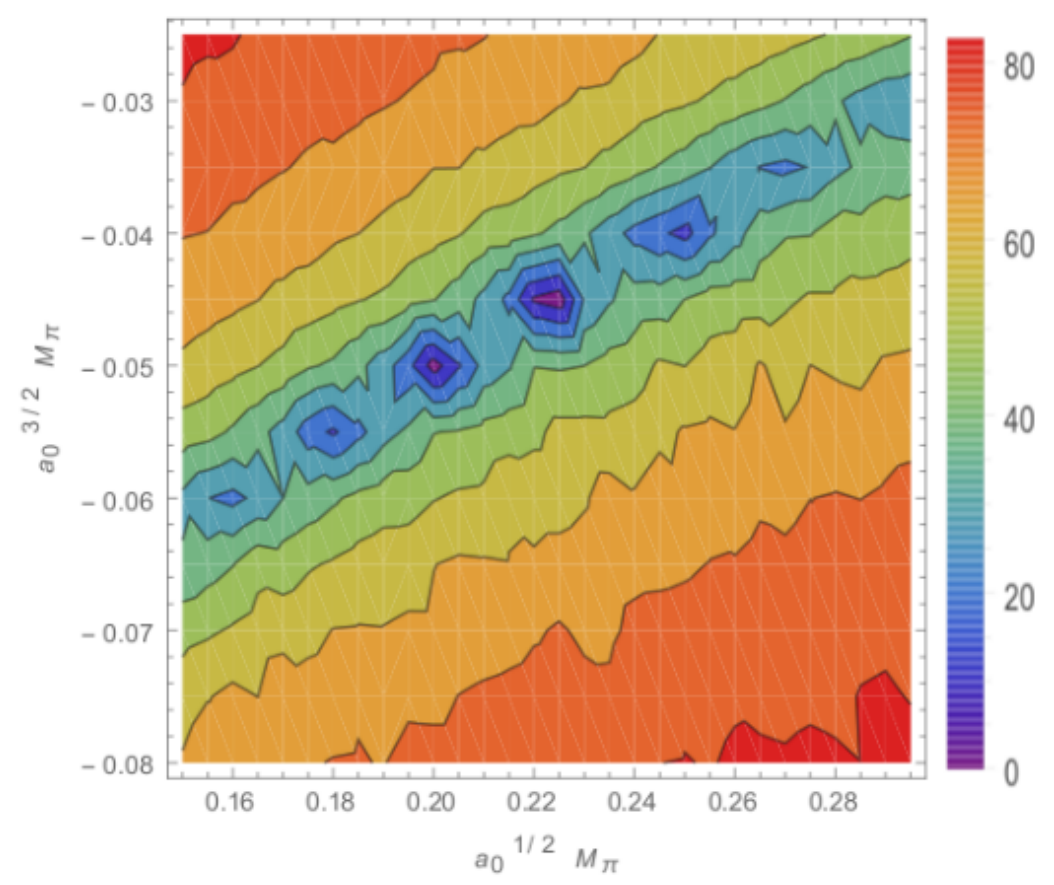

Figure 4: Value of the $\pi K \operatorname{RS} \chi$-like function defined in (2.1) for a grid of points on the $I=1 / 2, I=3 / 2$ scattering length plane. This result suggests that one can achieved an exact solution of the pion-kaon RS equations on the universal band.

\section{Acknowledgments}

We would like to thank the organizers for a wonderful workshop, and for the invitation to talk about our work on pion-pion and pion-kaon scattering. We are grateful to B. Moussallam for helful dicussions and to J. Bijnens for providing us the code for the NNLO pion-kaon scattering amplitudes. We also acknowledge useful discussions with M. Hoferichter, B. Kubis and U.-G. Meißner. Financial support by the Swiss National Science Foundation is gratefully acknowledged.

\section{References}

[1] M. Amaryan, U. G. Meißner, C. Meyer, J. Ritman and I. Strakovsky, arXiv:1804.06528 [hep-ph].

[2] S. Weinberg, Physica A 96 (1979) 327.

[3] J. Gasser and H. Leutwyler, Annals Phys. 158 (1984) 142.

[4] J. Gasser and H. Leutwyler, Nucl. Phys. B 250 (1985) 465.

[5] S. Weinberg, Phys. Rev. Lett. 17 (1966) 616.

[6] J. Bijnens, G. Colangelo, G. Ecker, J. Gasser and M. E. Sainio, Phys. Lett. B 374, 210 (1996).

[7] J. Bijnens, G. Colangelo, G. Ecker, J. Gasser and M. E. Sainio, Nucl. Phys. B 508, 263 (1997) Erratum: [Nucl. Phys. B 517, 639 (1998)].

[8] J. Bijnens and G. Ecker, Ann. Rev. Nucl. Part. Sci. 64, 149 (2014).

[9] J. R. Batley et al. [NA48-2 Collaboration], Eur. Phys. J. C 70, 635 (2010). 
[10] J. J. Dudek, R. G. Edwards and C. E. Thomas, Phys. Rev. D 86, 034031 (2012).

[11] Z. Fu, Phys. Rev. D 87, no. 7, 074501 (2013).

[12] L. Liu et al., Phys. Rev. D 96, no. 5, 054516 (2017).

[13] B. Ananthanarayan, G. Colangelo, J. Gasser and H. Leutwyler, Phys. Rept. 353 (2001) 207.

[14] G. Colangelo, J. Gasser and H. Leutwyler, Nucl. Phys. B 603, 125 (2001).

[15] R. García-Martín et al., Phys. Rev. D 83 (2011) 074004.

[16] R. Navarro Pérez, E. Ruiz Arriola and J. Ruiz de Elvira, Phys. Rev. D 91, 074014 (2015).

[17] V. Bernard, N. Kaiser and U. G. Meißner, Phys. Rev. D 43, 2757 (1991).

[18] V. Bernard, N. Kaiser and U. G. Meißner, Nucl. Phys. B 357, 129 (1991).

[19] S. Aoki et al., Eur. Phys. J. C 77, no. 2, 112 (2017).

[20] J. Bijnens and G. Ecker, Ann. Rev. Nucl. Part. Sci. 64, 149 (2014).

[21] J. Bijnens, P. Dhonte and P. Talavera, JHEP 0405, 036 (2004).

[22] P. Buettiker, S. Descotes-Genon and B. Moussallam, Eur. Phys. J. C 33, 409 (2004).

[23] T. Ledwig et al., Phys. Rev. D 90, no. 11, 114020 (2014), Phys. Rev. D 84, 096006 (2011).

[24] B. Adeva et al. [DIRAC Collaboration], Phys. Rev. D 96, 052002 (2017).

[25] S. M. Bilenky, V. H. Nguyen, L. L. Nemenov and F. G. Tkebuchava, Yad. Fiz. 10, 812 (1969).

[26] J. Schweizer, Phys. Lett. B 587, 33 (2004).

[27] J. Schweizer, Eur. Phys. J. C 36, no. 4, 483 (2004).

[28] B. Kubis and U. G. Meißner, Nucl. Phys. A 699, 709 (2002).

[29] J. M. Flynn and J. Nieves, Phys. Rev. D 75, 074024 (2007).

[30] S. R. Beane et al., Phys. Rev. D 74, 114503 (2006).

[31] Z. Fu, Phys. Rev. D 85, 074501 (2012).

[32] K. Sasaki et al. [PACS-CS Collaboration], Phys. Rev. D 89, no. 5, 054502 (2014).

[33] S. M. Roy, Phys. Lett. B 36 (1971) 353.

[34] I. Caprini, G. Colangelo and H. Leutwyler, Phys. Rev. Lett. 96, 132001 (2006).

[35] R. Garcia-Martin et al., Phys. Rev. Lett. 107, 072001 (2011); Phys. Rev. D 90, no. 9, 097901 (2014) Phys. Rev. D 93, no. 7, 076004 (2016).

[36] G. E. Hite and F. Steiner, Nuovo Cim. A 18 (1973) 237 [CERN-TH-1590 for app. D and E].

[37] J. Gasser and G. Wanders, Eur. Phys. J. C 10, 159 (1999).

[38] M. Hoferichter, J. Ruiz de Elvira, B. Kubis and U. G. Meißner, Phys. Rev. Lett. 115, 092301 (2015), Phys. Rept. 625, 1 (2016), Phys. Lett. B 760, 74 (2016) and J. Phys. G 45, no. 2, 024001 (2018). 